\title{
„Die Errettung der äußeren Wirklichkeit durch den Film“? Optische Erlösungsmomente im Werk Siegfried Kracauers zwischen Metaphysik und populärer Kultur
}

\author{
Carsten Heinze
}

Eingegangen: 16. August 2020 / Überarbeitet: 27. Juni 2021 / Angenommen: 5. Juli 2021 / Online publiziert: 30. Juli 2021

(C) Der/die Autor(en) 2021

Zusammenfassung Der Beitrag setzt sich mit dem Zusammenhang von Religion und Metaphysik auf der einen und Kino, Film und Fotografie als Ausdruck populärer Kultur auf der anderen Seite im Werk Siegfried Kracauers auseinander. Es wird gezeigt, dass Kracauers Zugänge zum Film und die Bedeutung, die er visuellen Medien wie der Fotografie und dem Film für die Kultur der Moderne zuschreibt, sich mit seinen frühen Arbeiten zu Metaphysik und Religion spannungsreich in Beziehung setzen lassen und dieses Spannungsverhältnis sich auch in seinen späteren Auseinandersetzungen mit dem Film fortschreibt. Dem Film werden verschiedene Funktionen, vor allem ein „Erlösungsmoment“, in diesem Spannungsverhältnis zugeschrieben, das vor dem Hintergrund seiner frühen metaphysischen Schriften $\mathrm{zu}$ verstehen ist. Es wird gezeigt, dass dieses „Erlösungsmoment“ auch in seinem letzten großen Werk, das sich der Geschichte widmet, noch einmal aufgegriffen, problematisiert und verarbeitet wird.

Schlüsselwörter Siegfried Kracauer · Filmtheorie $\cdot$ Medientheorie · Filmsoziologie · Religion und Film · Religion und Populärkultur 


\title{
„The Redemption of Physical Reality“ through Film? Visual aspects of salvation in Siegfried Kracauer's work between metaphysics and popular culture
}

\begin{abstract}
The article deals with the connection between religion and metaphysics as well as film and photography in Siegfried Kracauer's oeuvre. It argues that Kracauer's approaches to film and the importance he assigns to visual media such as photography and film for modern culture can be related to his early works on metaphysics and religion in a tense manner, and these tensions persist in his later engagements with the film. It is shown that the film is assigned various functions, especially the "redemption of physical reality", which are taken up and processed again in his last great work, which is dedicated to history.
\end{abstract}

Keywords Siegfried Kracauer - Film theory - Media studies · Religion and film · Popular culture and religion · Film sociology $\cdot$ Film studies $\cdot$ Media theory

\section{Einleitung}

Das 20. Jahrhundert gilt als Zeitalter des Films und des Kinos. In den ersten dreißig Jahren ihrer Existenz, so schreibt Geoffrey Nowell-Smith, sei die Ausweitung dieser populärkulturellen Unterhaltungsform durch ein Wachstum ,,beispiellosen Ausmaßes“ geprägt (2006, S. 3). Filme erzeugten seinerzeit eine massenmediale Anziehungskraft, und überall dort, wo sie gezeigt wurden, entstanden große und pompöse Filmpaläste, die in ihrer äußeren Architektur und ihren opulenten Innenräumen nicht selten an Kathedralen erinnerten. Aber anders als in den Zeremonien und Ritualen von Religionen, die auf stilles Gedenken, festen Zusammenhalt, inneres Seelenheil und gemeinschaftliche Erfahrung durch die spirituelle Verbindung mit einem Höheren abzielen, bietet das Kino, wie Siegfried Kracauer in einem Artikel für die Frankfurter Zeitung am 4. März 1926 über die Berliner Lichtspielhäuser schreibt, einen profanen „Kult der Zerstreuung“. Diese Zerstreuung sei als „Abbild des unbeherrschten Durcheinanders unserer Welt" zu verstehen und werde, so legt es der Artikel in seiner exemplarischen Kritik an der modernen Unterhaltungsindustrie nahe, nicht nur durch den Film als zusammenmontiertes Artefakt bewirkt, sondern sei bereits in der Architektur der großen Filmpaläste angelegt: „Gepflegter Prunk der Oberfläche ist das Kennzeichen dieser Massen-Theater. Sie sind, wie die Hotelhallen Kultstätten des Vergnügens, ihr Glanz bezweckt die Erbauung.“ (1977d [1926], S. 311). Der einzige Zweck dieser ,Zurüstung der Lichtspielhäuser“ sei es, ,das Publikum an die Peripherie zu fesseln, damit es nicht ins Bodenlose versinke." (ebd., S. 314) Auf diese Weise erklärt Kracauer das Kino zum säkularen Ort, das als Surrogat für den Verlust der Metaphysik dient und den Menschen in seiner unbehausten Existenz auffängt.

In diesem Zitat spiegelt sich aber auch der Grundtenor seiner Arbeiten wider, die sich durchgängig durch die Auseinandersetzung mit Glaubensfragen, Sinnsuche und Existenzbewältigungsstrategien auszeichnen, wobei Kracauer sich seit Mitte der 20er-Jahre immer stärker auf „dichte Beschreibungen“ (Geertz 2015 [1997]) 
massenkultureller Phänomene konzentriert und zu einem von den Arbeiten Georg Simmels inspirierten „Soziologen“ mutiert. Hierdurch rücken auch philosophische und theologische Bezugnahmen, die sein frühes Werk ausgezeichnet haben, in den Hintergrund: Sie markiert er jetzt als rein spekulative Vorgehensweisen, um an ihre Stelle eine funktionale Deutung von Religion zu setzen und einen fast schon empirisch zu nennenden Zugang zu jenen Kulturphänomenen zu suchen, die er als quasi-religiöse Surrogate identifiziert.

In seinen Schriften zum Film, aber nicht nur dort, lassen sich diese Bezugnahmen und Analogiebildungen immer wieder entdecken, sei es, dass er über Filme mit religiösem Inhalt schreibt, sei es, dass er Filme ideologiekritisch unter die Lupe nimmt, sei es, dass er den Film philosophisch als konkret-sinnliche Erfahrung in einer metaphysisch sinnentleerten und von der Religion entfremdeten Welt versteht sowie schließlich, dass er dem Film eschatologische Qualitäten in der materialen Errettung von der äußeren Wirklichkeit in seiner „Theorie des Films“ (1985) zuschreibt.

Man kann diese religiösen, mit Blick auf den Film apotheotisch zu nennenden Bezugnahmen bis in die Performativität seiner Sprach- und Begriffswahl hinein verfolgen, jedoch bleiben das Religiöse und Religion als solche bei ihm weitgehend unbestimmt. Offensichtlich ist Letztere in ihrer traditionellen Übermittlung, ihren rituellen Praktiken und Sinngebungsangeboten noch so alltagsweltlich verbreitet, dass sich Kracauer nicht um ihre Definition kümmern muss, eine Vorgehensweise, die er, wie Detlef Pollack zeigen kann, mit den Klassikern der Soziologie von Emile Durkheim über Max Weber bis hin zu Georg Simmel teilt (Pollack 2018, S. 17). Es reicht offensichtlich aus, assoziativ Formulierungen wie das Heilige, die Erlösung etc. aufzurufen, um die eigene Perspektive auf diese Zusammenhänge entfalten und plausibilisieren zu können. Diese richtet sich bei Kracauer nunmehr auf die Fragen, welche Sinnangebote in der Moderne an die Stelle von Religion treten, was diese mit Religion gemeinsam haben, aber auch, was sie von diesen unterscheidet.

Denn für Kracauer gibt es angesichts der mit Ratio durchwirkten modernen Gesellschaft kein Entkommen aus dem Verlust des Sinnes, den religiöse Ordnungen traditionell gewährt haben. So konstatiert er, dass sie zwar als Verlusterfahrung in der Gesellschaft erhalten bleiben, aber nicht länger dazu in der Lage sind, Antworten auf die Herausforderungen der modernen Welt zu liefern. Dies leiste jedoch u. a. der Film und sein Aufführungsort, das Kino. Somit gesteht er diesen quasi-religiöse und kultische Eigenschaften und dennoch Modernität zu.

Auch wenn sein Werk auf den ersten Blick wenig systematisiert und deshalb fragmentarisch erscheint, zumal es von breitgestreuten Sujets geprägt ist, lassen sich in ihm gedankliche Stränge und Verknüpfungen entdecken, die vor allem durch die Suche nach einem tieferen Sinn grundiert sind, den er hinter den Oberflächenerscheinungen der modernen Welt vermutet und in den optischen Oberflächen der modernen Kultur aufspürt. So heißt es programmatisch in seinem Aufsatz zum „Ornament der Masse“: „Der Ort, den eine Epoche im Geschichtsprozess einnimmt, ist aus der Analyse ihrer unscheinbaren Oberflächenphänomene schlagender zu bestimmen als aus den Urteilen der Epoche über sich selbst.“ (Kracauer 1977f [1927], S. 50). 
Im Folgenden geht es deshalb darum, Kracauers Einlassungen zum Zusammenhang zwischen Existenz- und Sinnfragen und dem Entstehen und der Entwicklung moderner Massenkultur, die er - seiner Zeit entsprechend - u. a. am Film, dem Kino und der Fotografie exemplifiziert, nachzuspüren und dabei den Fokus darauf zu richten, wie er Religion als Institution und Religiöses als Erfahrungsdimension in diesen Zusammenhang einbettet.

\section{Der Verlust metaphysischer Ordnung und innerer Stabilität - biografische und werkgeschichtliche Anmerkungen zu Siegfried Kracauer}

Wie viele Intellektuelle seiner Zeit war Siegfried Kracauer ein Agnostiker, der sich persönlich keiner Religion verpflichtet fühlte. Seine jüdische Herkunft ließ ihn zwar ein historisches Interesse für deren Kultur entwickeln; aber er teilte weder deren Glaubensüberzeugungen noch praktizierte er die dazugehörigen rituellen Handlungsvorschriften. Dennoch bezog sein Denken Inspirationen von der Theologie, etwa eines Søren Kierkegaards oder des Rabbiners Nehemias Anton Nobel, der einen Kreis junger jüdischer Intellektueller um sich scharte, dem neben Kracauer auch Leo Löwenthal angehörte (Wendt 2010, S. 89). Mit Löwenthal wird Kracauer eine lebenslange Freundschaft eingehen, über deren intellektuelle und emotionale Tiefe ein reger Briefwechsel Auskunft gibt (Jansen et al. 2003).

Als Konstante wird sich in Kracauers Denken der Wille zur Auseinandersetzung mit Religion und religiösen Deutungsangeboten erweisen, die von ihm fachkundig kommentiert werden, wobei Kracauer sich selbst als eine „sozial freischwebenden Intelligenz" begreift (Mannheim 1985 [1929], S. 135) und eine eigene weltanschauliche Positionierung verweigert: So schreibt er Kommentare und Rezensionen zur Bibelübersetzung, zum Katholizismus und zum Judentum und äußert sich konfessionsübergreifend zur religiösen Lage in Deutschland. Gleichzeitig verschiebt sich aber sein Blick auf Religion und Religiostät dahingehend, dass er sich von einer anfänglichen Selbstzentriertheit, in der er sich als Intellektueller begreift, der von „transzendentaler Obdachlosigkeit“" betroffen ist, löst und hin zu einer nach außen gerichteten gesellschaftstheoretischen Perspektive bewegt.

Früh richtete er sein Interesse auf die massenhaft verbreitete Kultur des Profanen, nimmt das Kino als Verbreitungsort des neuen Mediums Film, aber auch die Fotografie in den Blick, um an ihnen erkenntnis- und wissenstheoretische Fragestellungen auszuloten. Gleichzeitig führte er sie aber auch als Entitäten vor, aus denen sich exemplarisch das Wesen moderner Vergesellschaftung extrahieren lässt. Insbesondere das Kino und den Film beobachtete er dabei als massentaugliche Surrogate für den Verlust an religiösen Haltungen und religiösem Glauben, verlängerte so seine exemplarischen Analysen hin zu religionssoziologisch anmutenden Beobachtungen und Deutungen.

Um Siegfried Kracauers Bezugnahmen auf Kino, Film und Fotografie sowie seine filmtheoretischen Schriften im Hinblick auf religiöse Deutungsversuche genauer untersuchen zu können, soll zuerst ein kurzer biografischer, aber auch werkgeschichtlicher Abriss vorangestellt werden. 
Siegfried Kracauer wurde 1889 in Frankfurt am Main als Sohn einer jüdischen Tuchhändler-Familie geboren. In seinem Elternhaus und seiner Familie spielte die jüdische Religion weniger als religiöse Praxis, sondern eher in ihrer kulturellen Überlieferung eine Rolle (Brodersen 2001, S. 21 ff.). Er besuchte das „Philanthropien“ der Frankfurter Israelitischen Gemeinde, eine Reformschule, die den Idealen der Aufklärung verpflichtet war und an der auch sein Onkel Isidor Kracauer unterrichtete, ein berühmter Historiker der jüdischen Geschichte, der ihn dort zum ersten Mal inhaltlich mit ihr in Berührung brachte.

Kracauer hatte einen Sprachfehler, er stotterte, und infolgedessen eine schwierige Kindheit; sein Stottern verhinderte später auch eine Lehrtätigkeit an einer Universität. Nach dem Abitur 1907 nahm er das Studium der Architektur an der Technischen Universität Darmstadt auf, das er mit einem Diplom abschloss. Anschließend promovierte er in Berlin mit einer kunsthistorischen Thematik über die Schmiedekunst in Preußen.

Schon während seines Architekturstudiums, das dem Wunsch seiner Eltern entsprach, die sich für ihren einzigen Sohn eine sichere Berufsperspektive wünschten, studierte er Philosophie und Soziologie in Darmstadt, Berlin und München, wobei ihn insbesondere die Vorlesungen Georg Simmels an der Friedrich-Wilhelms-Universität zu Berlin, der heutigen Humboldt Universität, beeindruckten. In der Folge wird Kracauer Simmel seine Seminararbeiten zusenden und um Korrekturvorschläge bitten, eine Bitte, der Simmel mit „kritischen Beurteilungen“ (Wendt 2010, S. 97) nachkommt. Obwohl Kracauer seit 1910 auch als Architekt arbeitete, verfolgte er dennoch eine andere, sozial- und kulturwissenschaftliche geprägte Laufbahn, wofür seine 1914, kurz vor Beginn des Ersten Weltkriegs, abgeschlossene Promotion steht.

Der Erste Weltkrieg markierte, wie bei anderen jungen Männern seiner Generation auch, ein abruptes Ende seiner langfristigen beruflichen Pläne. Kracauer, der sich freiwillig zum Wehrdienst meldete - seine Bewerbung wurde jedoch zunächst zurückgestellt und er stattdessen für den heimischen Sanitätsdienst rekrutiert -, verlor rasch die anfängliche Begeisterung für den Krieg. Unbeirrt suchte er weiter nach intellektuellen Auseinandersetzungen, u.a. mit Max Scheler, den er 1916 kennenlernte, und arbeitete an verschiedenen Publikationen, die er vor allem nach 1920 in großer Regelmäßigkeit veröffentlichte.

Ab 1921 arbeitete er im Feuilleton der Frankfurter Zeitung, eine Tätigkeit, die er bis zu seiner Kündigung im August 1933 fortführte. In den frühen 20er-Jahren begann aber auch seine Freundschaft mit dem wesentlichen jüngeren, zum damaligen Zeitpunkt Noch-Schüler Theodor. W. Adorno, den er intellektuell fördern sollte, und mit Leo Löwenthal. Beide wurden vor 1933 zu berühmten Protagonisten der sogenannten „Frankfurter Schule“, Adorno als Professor für Soziologie an der „Johann Goethe Universität" und Leo Löwenthal als Wissenschaftler am neu gegründeten „Institut für Sozialforschung“"

In den frühen 20er-Jahren verband diese drei aber nicht nur eine innig zu nennende Freundschaft, sondern eine Seelenverwandtschaft, die sie dazu veranlasste, von sich als „Hauptquartier des Wohlfahrtsquartiers für transzendentale Heimatlose" (Löwenthal 2003, S. 272) zu sprechen, so Adorno und Kracauer in einem Brief an Löwenthal. Und diese Formulierung der transzendental Heimatlosen (an anderer Stelle auch Obdachlosen), die sie, wie Kracauer bekennt, ihrem „Kultbuch“, nämlich 
Georg Lukàcs’ 1920 auf Deutsch veröffentlicher „Theorie des Romans“, entnommen haben, gibt metaphorisch das sich für Kracauer herausbildende Lebensgefühl wieder, das nun auch zum Prä seiner erkenntnistheoretisch orientierten Schriften und seiner Suche nach weltlichen statt religiösen Transzendenzphänomenen werden sollte.

Während sich die Freundschaft zwischen Kracauer und Adorno nicht zuletzt aufgrund persönlicher Empfindlichkeiten im Laufe der 20er-Jahre abkühlte, hielt sie zu Leo Löwenthal ein Leben lang. Kracauer war es auch, der Löwenthal mit Ernst Bloch und Max Horkheimer bekannt machte, die zur damaligen Zeit zur Frankfurter Intellektuellenszene dazugehörten, später dann wichtige Repräsentanten der Frankfurter Schule wurden.

In den inhaltlichen Auseinandersetzungen in dieser Intellektuellenszene wurde durchaus mit harten Bandagen gekämpft, wobei Kracauer über eine besonders effektive Kritikform verfügte: Rezensionen über die Werke der anderen in einem der wichtigsten Publikationsorgane der Weimarer Republik, der Frankfurter Zeitung. Jedenfalls scheinen das Beleidigen (über inhaltliche Kritik) und das Beleidigtsein darüber zu entscheiden, ob sich die Beziehungen zwischen diesen Intellektuellen als widerstandsfähig erwiesen, bis hin zur Frage, ob man bereit war, einen der anderen bei der Stellensuche, die im akademischen Bereich damals wie heute einem „Hasard“ (Weber 1996 [1919], S. 15) gleicht, zu unterstützen oder gar zu protegieren.

Inhaltlich wandte sich Kracauer als genauer Beobachter seiner Zeit gegen den Messianismus jüdischer Intellektueller, den er sowohl in den säkularen Ausformungen, beispielsweise des Marxismus und des national orientierten Zionismus, als auch in den theologisch-religiösen Arbeiten eines Bubers oder Rosenzweigs ausmachte. So entwarf er auch bezüglich der Weltanschauungslehre des Marxismus eine eigene Marx-Interpretation (Frisby 1989, S. 132).

Anders als bei vielen seiner Zeitgenossen entstand bei Kracauer sehr früh eine Leidenschaft und ein Verständnis für die neue populäre Kultur, die er immer wieder in diversen Zeitungsartikeln, Rezensionen und Essays thematisierte. Als er Ende 1933, nach der Kündigung seiner Stelle als Leiter der Feuilleton-Redaktion der Frankfurter Zeitung, die in Berlin ansässig war, nach Paris floh, waren es mehr als 2000 Artikel, die er in den 12 Jahren bei der Frankfurter Zeitung publiziert hatte, darunter die zuerst als Serie erschienene Studie über „Die Angestellten. Aus dem neuesten Deutschland“ (Kracauer 2017 [1930]), die ein Meilenstein in der sozialwissenschaftlichen Erforschung einer sich (damals) neu konstituierenden Gesellschaftsschicht ist.

Diese Serie war so erfolgreich, dass sie bereits 1930 als Buch erschien. In ihr konstatiert Kracauer einen Widerspruch zwischen der in materieller und sozialer Hinsicht proletarisierten Lage dieser Schicht und ihrem weiterhin von bürgerlichen und mittelständischen Werten geprägten Bewusstsein, diagnostiziert bei ihr eine kollektive „Obdachlosigkeit“ und einen ausgeprägten Eskapismus hin zu den Angeboten der Kulturindustrie: dem Film, den Revuen, dem Schlager, den Operetten und Tanzlokalen. So spannt er einen Bogen von ,Sensationsmilieus mit inniger Affektivität zum Spektakel bis hin zu den Arbeitswelten, in denen das Gegenteil gefragt ist: nämlich völlige Unauffälligkeit“ (Matala de Maza 2018, S. 17). Als Journalist eine soziologische Perspektive einzunehmen, einen langen Atem bei der Beobach- 
tung gesellschaftlicher Phänomene zu entwickeln, ein fast schon ethnografisches methodisches Vorgehen (Interviews, Fotos, Beobachtungsprotokolle, Dokumentenauswertung) ohne Vorbilder zu praktizieren und das Entdeckte theoretisch durchaus anspruchsvoll zu untermauern, es aber zugleich durch dichte Beschreibungen diverser Alltagsphänomene plausibel zu machen: das ist ein Verdienst, das Kracauer spätestens mit dieser Studie zukommt und ihn als den öffentlichen Soziologen ${ }^{1}$ der späten Weimarer Republik ausweist. Am 10. Mai 1933 gehörte „Die Angestellten“ zu den Büchern, die insbesondere von Mitglieder*innen des NS-Studentenbundes auf Scheiterhaufen verbrannt wurden, um sich - wie sie aggressiv verkündeten „undeutscher Literatur“ ein für alle Mal zu entledigen (https://www.bpb.de/politik/ hintergrund-aktuell/268884/ns-buecherverbrennung, Stand: 20/06/2021).

Bereits 1930 war Kracauer von der Frankfurter Zeitung wegen seiner Fähigkeit, mit kritischen Gesellschaftsdiagnosen ein breites Publikum zu interessieren, aber auch politische Verwerfungen, beispielsweise die wachsende kulturelle Stärke der Nazis und ihr Einsickern in bürgerliche Kreise, aufzudecken, nicht zuletzt aber auch wegen seines Jüdischseins gemaßregelt und nach Berlin zwangsversetzt worden, „wo man ihn mit Gehaltskürzungen, der zunehmenden Ablehnung seiner Artikel und der Aufforderung, sich einen Nebenerwerb zu suchen“ (Wendt 2010, S. 88) demütigte.

Wie viele jüdische Intellektuelle, auch seines inneren Zirkels, floh er 1933 erst über Paris, dann über Marseille und Lissabon in die USA. Dort wurde er wissenschaftlicher Mitarbeiter der „Film Library des Museums of Modern Art“, zudem erhielt er immer wieder Stipendien, mit deren Hilfe er seine nunmehr auf Englisch erscheinenden Forschungsarbeiten weiterführen konnte. Sein Einkommen blieb nach seiner Flucht zeitlebens prekär. Kracauer war somit gegen Ende seines Lebens sowohl in materieller, geografischer als auch soziokultureller Hinsicht zwar ,obdachlos“ geworden, sein Biograf Momme Brodersen bezeichnet diese Phase treffend ,im Niemandsland der Exterritorialität" (Brodersen 2003, S. 120 ff.), aber nicht in mentaler und inhaltlicher Hinsicht. So scheint Kracauer auf der persönlichen Ebene seinen inneren Frieden mit dieser Vorläufigkeit und Nicht-Auflösbarkeit des Zustands eines Wartenden geschlossen zu haben, ohne einer konfessionellen oder ideologischen Glaubensrichtung als Sinnstiftung nachgegeben zu haben.

Obwohl er Deutschland nach dem Zweiten Weltkrieg, anders als Adorno und Horkheimer, nicht mehr als Lebensmittelpunkt ansah und bis zu seinem Tod in den USA blieb, führten ihn ausgedehnte Reisen nach Europa und Deutschland. 1964 war er Teilnehmer des Kolloquiums „Poetik und Hermeneutik“ in Köln, ebenso 1966 in Lindau, bei dem prominente Wissenschaftler wie Hans Blumenberg, Odo Marquard und Karlheinz Stierle zugegen waren. Dort konnte er seine Thesen zur Geschichte vorstellen. Am 26. November 1966 erlag Siegfried Kracauer in New York einer Lungenentzündung.

\footnotetext{
1 Der amerikanische Soziologe Michael Burawoy forderte die Soziologie und die Soziolog*innen angesichts der Dominanz der Ökonomik im öffentlichen Diskurs dazu auf, endlich wieder die Soziologie als gesellschaftskritische Stimme ins Spiel zu bringen (Burawoy 2015, S. 23 ff.). Seine Rede von der Public Sociology hat auch in der zeitgenössischen Soziologie Deutschlands einen großen Widerhall gefunden (Neun 2018).
} 
Ein Blick in die Werkgenese zeigt, dass sich Kracauer in seinen frühen Schriften, nicht zuletzt aufgrund persönlich empfundener Einsamkeit und sozialer Isoliertheit, mit philosophischen Erkenntnisfragen beschäftigt hat, indem er sich kritisch mit dem religiösen bzw. metaphysischen Sinnverlust in der Moderne auseinandersetzt, der für ihn zugleich ein Ende der ursprünglichen menschlichen Gemeinschaft bedeutet. In seiner Arbeit „Von der Erkenntnismöglichkeit seelischen Lebens“ aus dem Jahr 1916 stellt er die Erforschung der ewig gleichbleibenden Natur dem jeglichen Halt entbehrenden Prozess des Lebens als dynamischen Gegenstand der Geisteswissenschaft gegenüber. Die Naturwissenschaften tauchen darin als eine antimetaphysische Wissenschaft auf, die die ,unbeseelte Natur“ zum Gegenstand hat und ,auf dem Glauben an die gesetzmäßige Abfolge der innerhalb der Materie stattfindenden Veränderungen“ aufbaut (2004a, S. 124). Trotz einiger Parallelen zur Lebensphilosophie bleibt Kracauer zu ihr auf Distanz. Aus den Betrachtungen der Möglichkeit und der Grenzen des Erkennens zieht er den Schluss, dass absolute, auf eine „höchste Wahrheit““ ausgerichtete Erkenntnis nicht mehr möglich sei: „Es gibt kein Ende im Erkennen, kein letztes Ziel, das einem endgültigen Abschluß gleichkäme; so unerschöpflich, wie das Leben selber ist, sind auch die Möglichkeiten seiner begrifflichen Ergründung." (ebd., S. 165).

In dem Artikel „Autorität und Individualismus“ aus dem Jahr 1921 konstatiert Kracauer (2011 [1921], S. 167 ff.) aufgrund der vorherrschenden Weltanschauungsgegensätze chaotische gesellschaftliche Zustände, die sich sowohl in seinen persönlichen Lebensumständen als auch den äußeren Zeitumständen der frühen Nachkriegsjahre widerspiegeln (Brodersen 2001, S. 29). Die Lösung dieses gesellschaftlichen Dilemmas sieht er in einer auf Autorität begründeten Gemeinschaftsform, die er der sich gerade konstituierenden Demokratie in Deutschland geradewegs empfiehlt. An die ,charismatische Herrschaft“ Max Webers erinnernd, schreibt Kracauer, dass es einer natürlichen Autorität bedürfe, um zu einer ,traditionellen Gebundenheit des Lebensstils“" zurückzufinden (2011 [1921], S. 172).

Dieser Sinnverlust in der Moderne ist nach Kracauer vor allem auf die methodischen Abstraktionen der modernen Wissenschaft und der dadurch erzeugten Entleerung der Wirklichkeit durch quantitative Forschung sowie auf die Durchsetzung einer kapitalistischen, in sich selbst erschöpfenden Rationalität zurückzuführen. In dieser Schaffensphase bewegt sich Kracauer zwischen spezifischen Lesarten des Marxismus und der Theologie, deren Kombination er besonders in den Arbeiten Ernst Blochs zu schätzen weiß (Frisby 1989, S. 131 f.).

So wirft er Max Scheler in seiner Rezension zu „Probleme der Religion. Zur religiösen Erneuerung“ (1921) Standpunktlosigkeit und ein verhohlenes missionarisches Eintreten für den Katholizismus vor. Es ist jedoch weniger der Vorwurf der Prinzipienlosigkeit gegenüber Scheler, der hier interessiert, als die von Kracauer aufgeworfenen Fragen, mithilfe derer er sich letztlich selbst zu charakterisieren scheint: „Wer kennt ihn nicht, den Intellektuellen von heute, der, weil er es im Vakuum der Glaubenslosigkeit nicht mehr aushält, auf Grund romantischen Willensentschlusses irgendwo Unterschlupf sucht? Ihm vielleicht wird durch Scheler der Weg hinüber zum Katholizismus geebnet. Menschen dieser Art aber, die solchen Schleichweg der Schwäche, wenn auch einer sehr begreiflichen Schwäche, wandeln, sind gewiß nicht die Besten; begehren die doch voreilig Erfüllung ihrer kurzatmigen Sehnsucht, statt 
im Vakuum tapfer auszuharren und zu - warten.“ (Kracauer 1977a [1921], S. 194). Seine Kritik an Scheler verlängert er zu einer Skizzierung der allgemeinen religiösen Lage in Deutschland und zur Rolle und Position der Intellektuellen, wenn er schreibt: „Die Gestalt Schelers, wie sie aus diesem Werk mit allen ihren Schwächen und Vorzügen hervorleuchtet, ist in vieler Hinsicht charakteristisch für unsere Zeit. Verzweiflung über Gottesentfremdung paart sich in der Gegenwart mit einer gegen früher ungemein gesteigerten religiösen Bedürftigkeit. Die Menschheit von heute drängt danach, einen Zugang zum religiösen Glauben zu finden und vermag doch im allgemeinen dieses Ziel nur mit den Mitteln eines Denkens zu erreichen, das mehr den Willen zum Glauben als den Glauben selber bekundet. Wurzellos, wie wir noch sind, können wir dem Relativismus kaum entrinnen und schweifen ruhelos von Erscheinung zu Erscheinung, von Kultur zu Kultur, uns in Ermangelung eigenen Seins in das Sein eines jeden Phänomens versenkend.“ (ebd., S. 196).

Eine Diskussion über religiöse Deutungsangebote findet sich auch in seiner Rezension zu Martin Bubers ,Ich und Du“, dem er ein Sendungsbewusstsein vorwirft, das die Vernachlässigung der konkreten Wirklichkeit zugunsten einer allumspannenden Sphäre des Religiösen hintertreibe (1990a [1923], S. 238). Auch in seiner weltanschaulichen Vermessung der "religiösen Lage in Deutschland“, in der er die zeitgenössischen Antworten des Marxismus dem Protestantismus und Katholizismus gegenüberstellt, vermag ihn keine der angebotenen Deutungen zu überzeugen; vielmehr sieht er ihnen ein Festhalten beziehungsweise Zurückfallen hinter alte Glaubenssätze (1990b [1924], S. 273 ff.). Jedoch schließt Kracauer nicht aus, dass etwas Kommendes die aufgerissene Lücke zu schließen in der Lage ist.

Die Soziologie als damals recht junges Fach, zu der Kracauer in seiner 1922 erschienen Studie „Soziologie als Wissenschaft“ (1971) Stellung bezieht, stellt für ihn die notwendige, an diesen Befunden ansetzende Wissenschaft der modernen Gesellschaft dar, die allerdings aufgrund ihres an Gesetzmäßigkeit und Regelmäßigkeit orientierten „Formalismus“, den er bei Max Weber und Georg Simmel zu entdecken glaubt, nicht zu den entscheidenden Sinnfragen als überwölbendes Dach des Gesellschaftlichen vorstoßen könne. Gleichzeitig aber war sich Kracauer bei aller Kritik der Unwiederholbarkeit einer solchen vormodernen Lebensform im Klaren.

Sein in dieser Werkperiode ambivalentes Verhältnis zur Moderne lässt ihn in die Position des „Wartenden“ rücken, der auf Erlösung hofft, der sich jedoch nicht voreilig unter ein vermeintlich sinnstiftendes Dach stellen will. So diagnostiziert Kracauer im gleichnamigen Artikel „Die Wartenden“ von 1922: „Horror vacui der Schrecken vor der Leere beherrscht diese Menschen. Und man begreift leicht, nach welchen Richtungen sich ihr Sehnen erstreckt. Alles in ihnen und um sie drängt auf erneutes Sein in der religiösen Sphäre und zugleich auf die Erlösung aus ungehemmter Freizügigkeit durch das Eingehen in die formgebende Gemeinschaft hin.“ (1977b, S. 109).

Die Wartenden sind die Versinnbildlichung der menschlichen Existenz in der Moderne; sie sind zu Wartenden geworden, weil sich religiöser Sinn, der traditionell mentale Sicherheit gewährt hat, substantiell entleert hat, weil sie aus den religiösen Gemeinschaften „,vertrieben“ und deshalb „vereinzelt“ sind und weil ihr „Relativismus" (ebd., S. 106f.) sie jedwedes Sinnangebot in Frage stellen lässt. In fast schon soziologischer Manier komplementiert Kracauer das Ensemble der Wartenden, die 
er als die zeitgemäßen Typen per se ansieht, um den „Skeptiker“ und den „Kurzschlussmenschen“. Und noch einmal skizziert er den Wartenden als jemanden, der sich zur konkreten Wirklichkeit hin öffnet und deshalb von ,,angespannter Aktivität und tätigem Sichbereiten“ (ebd., S. 118) angetrieben wird.

Die Auseinandersetzung mit der modernen populären Kultur, die Kracauer in den 1920er-Jahren beginnt, führen ihn früh zum literarischen Genre des Detektivromans (2006 [1922-25]). Seine Genre-Analyse, als philosophisches Traktat konzipiert, die im Anschluss an seine Auseinandersetzung mit der Soziologie erfolgt, ist insofern von besonderer Bedeutung, da er hier zum ersten Mal eine literarische Ausdrucksform der populären Kultur mit existentiellen Fragen verknüpft und zu einer kritischen Gesellschaftsdiagnose verdichtet.

Alle auftretenden Personen und Orte haben nach Kracauer im Detektivroman ihren vorherbestimmten Platz, der in unterschiedlichen gesellschaftlichen Sphären situiert ist, sodass die Personen die Lebenswelt des jeweils anderen nicht länger kennen können. Lediglich über die Figur des Detektivs vermittelten sich die verschiedenen Schichtungen (Frisby 1990, S. 46). Derart mutiert der Detektiv zum säkularen „Detektiv-Gott“, und „,der Anspruch seiner ratio auf Autonomie macht den Detektiv zum Widerspiel Gottes selber“ (Kracauer 2006 [1922-1925], S. 143). Es handelt sich bei seinem Wissen um eine säkulare Allwissenheit, die den einsamen, zwischen den Sphären wandelnden Detektiv auszeichnet - eine Allwissenheit, die gegenwärtig bleibt und Transzendenz leugnet (ebd., S. 142). Der „DetektivGott“ herrscht über eine Welt, ,die Gott verlassen hat“, er ist Herrscher des „Wesenlosen“ (ebd., S. 143), und seine „Erlösungstat“ besteht in der „Auflösung des aus ihr gewordenen Etwas“ (ebd., S. 141).

Kracauer vergleicht die Hotelhallen als Orte detektivischer Tätigkeit mit Gotteshäusern, um zu verdeutlichen, dass dort Gemeinschaften nicht nur zu unterschiedlichen Zwecken, sondern auch in unterschiedlichen Figurationen zusammenkommen: „Gilt aber das Gotteshaus dem Dienste dessen, zu dem man sich in ihm begibt, so dient die Hotelhalle allen, die sich in ihr zu niemandem begeben." (ebd., S. 130) Während das Gotteshaus als Ort der Vergemeinschaftung einem höheren Zweck dient und zur emotionalen Vergemeinschaftung beiträgt, zeichnen sich die Begegnungen in der Hotelhalle durch Indifferenz aus, erlaubt sie sowohl legale als auch illegale Handlungen, da sie Belanglosigkeit symbolisiert (ebd., S. 138f.). Das gesamte Interieur des Gotteshauses drückt das Bestreben zur Spannung nach einem Höheren aus, die Hotelhalle dagegen ist lediglich zivilisierter Ausdruck ihrer selbst (ebd., S. 132f.). Im Detektivroman entdeckt Kracauer somit nicht nur ein Substitut für Transzendenzerfahrungen, gar die Projektion der Figur des Detektivs auf das Gottesprinzip, weil dieser allwissend in einer Gesellschaft ist, deren GesamtStruktur opak geworden ist und nicht länger aufgrund von Erfahrungen erschlossen werden kann. Es ist diese Einsicht, die auch von ihm verlangt, nicht länger als Wartender aufzutreten, der auf eine gemeinschaftsstiftende Alternative zur Religion ebenso wie die übrigen hofft, sondern als distanzierter Beobachter gesellschaftlicher Zustände, deren Strukturmerkmale er in allererster Linie in Alltagsphänomenen und kulturellen Ausdrucksformen zu dechiffrieren sucht. Religion und deren kulturelle Ausdruckformen dienen ihm ab jetzt ausschließlich als Vergleichs- und Kontrastfo- 
lie, wie beispielsweise die Hotelhalle zum Gotteshaus, um die Spezifika moderner Transzendenz- und Religionssubstitute herauszuarbeiten.

In der zweiten Hälfte der 20er-Jahre beginnt Kracauer mit der Produktion unzähliger Essays, in denen er sich dem scheinbar Oberflächlichen widmet und Alltagsphänomene in den Mittelpunkt rückt, die vom Sport, den Tanzpalästen, Operetten und Revuetheatern über Straßen und Stehbars hin zu Kinos und dem Film reichen und den Menschen als Zerstreuung, also zu seelischen Entlastung dienen. Zugleich kritisiert er, dass die Orte der Zerstreuung, wie beispielsweise das Kino, einen Prunk und eine vermeintliche Ganzheitlichkeit vorgaukeln, die nichts mit dem realen Leben gemein haben, das zwischen Vermassung in der Arbeitswelt, in der Stadt und in der Freizeit bei gleichzeitiger Vereinzelung in der für den Einzelnen größtenteils anonym bleibenden Masse oszilliert.

So beschreibt Kracauer die Kinos als prunkhafte Orte der Zerstreuung, als „Kultstätten des Vergnügens“ und damit der Hotelhalle vergleichbar (Kracauer 1977d [1926], S. 311). Ihre Architektur symbolisiere eine nicht mehr vorhandene Einheit, die von einer Bewusstwerdung der eigenen Vermassung und Zerstreuung ablenke und damit einen ,falschen“ Zusammenhang vorgaukele: „Die Zerstreuung, die sinnvoll einzig als Improvisation ist, als ein Abbild des unbeherrschten Durcheinanders unserer Welt, wird von ihnen mit Draperien umhängt und zurückgezwungen in eine Einheit, die es gar nicht mehr gibt. Statt zum Zerfall sich zu bekennen, den darzustellen ihnen obläge, kleben sie die Stücke nachträglich zusammen und bieten sie als gewachsene Schöpfung an.“ (ebd., S. 316) Damit verschiebt Kracauer die Frage nach der fehlenden metaphysischen Sinntiefe und die Suche nach Zerstreuung in den Bereich der Populärkultur und erkennt in ihr, bei aller Kritik an ihrem häufig illusorischen Charakter, das Potenzial zur Selbstbewusstwerdung.

Seine Arbeiten zu Fotografie, Film und Geschichte werden ab jetzt Referenzpunkte seines Werks bleiben, auf die Kracauer auch bis zum Ende seines Schaffens immer wieder zurückkommen wird. Dass Kracauer selbst derartige Kontinuitäten in seinen Arbeiten erkannte, wird im letzten veröffentlichten Werk deutlich. So schreibt er in der Einführung seines posthum erschienen Geschichtsbuches „Geschichte - vor den letzten Dingen“: , ,[...] Ihrem [dem Beispiel autobiografisch reflektierender Historiker in Vorreden, C. H.] folgend darf ich wohl erwähnen, wie ich kürzlich unvermutet entdeckte, daß mein Interesse an der Geschichte - das sich vor etwa einem Jahr geltend machte und von dem ich bislang annahm, es sei durch den nachhaltigen Eindruck unserer gegenwärtigen Situation auf meinen Intellekt hervorgerufen - eigentlich aus den Ideen hervorging, die ich in meiner, Theorie des Films ‘ auszuführen suchte. Indem ich mich der Geschichte zuwandte, führte ich nur Gedanken fort, die in jenem Buch manifest waren." (1973, S. 15) Und nur wenige Zeilen später heißt es: „Blitzartig wurden mir die vielen Parallelen klar, die zwischen der Geschichte und den photographischen Medien, historischer Realität und Kamera-Realität bestehen. Vor kurzem stieß ich auf meinen Aufsatz zur ,Photographie“ und war völlig überrascht festzustellen, daß ich den Vergleich von Historismus und Photographie bereits in diesem Artikel aus den zwanziger Jahren gezogen hatte." (ebd.).

Durch diese Bemerkungen schließt sich der Themenkreis in Kracauers Werk, denn mit Blick auf den Vorraum der Geschichte ist zugleich eine metaphysische Grenze erreicht, die von der Einsicht geprägt ist, nicht mehr zu den „letzten Dingen“ vor- 
dringen zu können: Fotografie und Film bewahren die Welt zwar an ihrer Oberfläche, lassen aber keine Letztbegründungen und tieferen jenseitigen Sinnschichten mehr zu. Von ihnen geht keine Erlösung aus, sondern von ihnen ist allenfalls (momentane) Errettung durch Vergegenwärtigung zu erwarten.

So lässt sich bis hierher festhalten: Es zeigt sich in Kracauers Werk eine Kontinuität in der Wahl von Beschreibungsfiguren und performativer Sprache, indem er den modernen Menschen in eine exponierte Stellung rückt, die von „Leere“ und „Unbehaustheit“" und von der Suche nach den ,letzten Dingen“ geprägt ist. Da es für ihn weder eine Rückkehr zur vormodernen Ordnung noch eine moderne Variante der Metaphysik oder des Glaubens zu geben scheint, sucht er diese Lücke durch die Einlassung in die populäre Kultur zu füllen. Und er erkennt in ihr, bei aller Kritik an ihren teilweise Illusionen vorgaukelnden Ausdrucksformen, durchaus positive, gar emanzipatorische Züge.

\section{Fotografie und Film als trügerische Oberfläche oder Erlösung?}

Indem Kracauer die visuellen Medien Fotografie und Film als die vorherrschenden Ausdrucksformen moderner Kultur begreift, nimmt er den zu Beginn der 1990erJahre in den Kultur- und Sozialwissenschaften eingeläuteten ,,pictorial turn“2 vorweg. Seine durchgängig vertretene These, dass die Gesellschaftsbeschreibung der Moderne unzureichend sei, wenn sie nicht ihrer im Wesentlichen optisch geprägten kulturellen Ausdrucksformen gerecht würde, steht für diesen Perspektivenwechsel. Das „Primat des Optischen“ (Später 2016, S. 195 ff.), das Kracauer nicht nur auf die visuellen Medien beschränkt, sondern auf sämtliche kulturellen Oberflächenphänomene und ihre zeiträumlichen Organisationsformen bezieht, kulminiert in einer technikbasierten Wahrnehmungstheorie (die vom Ansatz her Walter Benjamins Medientheorie nahesteht). Gertrud Koch fasst diesen Ansatz wie folgt zusammen: „Das fotografische und das filmische Bild werden in einer dreistelligen semiotischen Operation fixiert: Die unendliche Mannigfaltigkeit der physischen, materialen Welt ist das Reservoir an Referenzobjekten, auf das sich Wahrnehmung bezieht. Und zwar in einer doppelten Operationalisierung: einer technischen durch die Rahmenbedingungen der Kamera (Linsen, Film, Lichtempfindlichkeit etc.) und einer personalen durch die sensorischen, kulturellen und psychischen Dispositionen des Wahrnehmungsapparats.“ (2012, S. 130) Mit Walter Benjamin stimmt Kracauer darin überein, dass Fotografie und Film in der Lage sind, die Welt auf eine spezifische Weise sichtund damit wahrnehmbar zu machen. ${ }^{3}$ Beiden kommt das Verdienst zu, technische Medien des Visuellen in einem kulturumfassenden Sinne thematisiert und ihre gesellschaftlichen Funktionen analysiert zu haben. Gleichzeitig ist beiden aber auch

\footnotetext{
2 Der amerikanische Kunsthistoriker William T. J. Mitchell gilt als derjenige, der - eine Formulierung Robert Rortys aufgreifend - die piktorale Wende einfordert, indem er darauf aufmerksam macht, dass das 20. Jahrhundert von einer Bilderwelt statt einer Textwelt überflutet worden ist (2007 [1994], S. 25 ff.).

3 Für eine Gegenüberstellung des ,utopischen Diskurses“ in den Medientheorien von Kracauer und Benjamin, siehe Butzer (2009, S. $153 \mathrm{ff}$.)
} 
die Anfälligkeit der Massenmedien und des Films für propagandistische Zwecke bewusst.

In Kracauers Schriften lässt sich im Hinblick auf den Film als optische Medialisierungsform und dessen Zusammenhang mit Religion und Religiösem folgende Unterscheidung finden: Entweder untersucht er Religion und religiöse Elemente als Gegenstand des Films oder er verallgemeinert diesen Zusammenhang, um ihn exemplarisch in gesamtgesellschaftliche Beschreibungen einzubetten.

Bereits in einer frühen Rezension aus dem Jahr 1916 geht Kracauer (2004b [1916], S. 12f.) auf einen Film mit religiösem Inhalt unter dem Titel „Die heilige Geschichte im Film“ ein. In ihr steht der italienische Film „Christus“ von Giulio Antamoro im Mittelpunkt. Nach einer skeptischen Vorrede hinsichtlich des Bedarfs nach derlei „Anfachung der Frömmigkeit“" bespricht er den Film wohlwollend und lobt seine visuelle Anschaulichkeit, die sich aus dem Dreh an Originalschauplätzen in Ägypten und Palästina ergebe.

In drei weiteren Rezensionen der Jahre 1924 (2004c; 2004d) und 1925 (2004e), die sich - für Kracauer ungewöhnlich - allesamt auf denselben Film, nämlich ,Die Straße“ von Karl Grune aus dem Jahr 1923, beziehen, treten die Verbindungen von Religion, Sinnfragen und erzählerischer Darstellung noch deutlicher hervor. Kurz zusammengefasst, geht es in dem Film um einen Ehemann, der sich zu den abendlich-verführerischen Lichtern der Straße mit all ihren Verlockungen und Gefahren hingezogen fühlt und diesem Verlangen nachgibt. Er erfährt das ,sinnlose verlockende Durcheinander des taumelnden Lebens“, das „Abenteuer“ und ,unausgekostete Möglichkeiten“ verspricht. Er gerät an eine Prostituierte, der er in ein Lokal folgt. Später wird er Zeuge eines Raubmordes, gerät jedoch durch seine Anwesenheit schnell in Verdacht und wird eingesperrt. Nur die Aussage eines Kindes rettet ihn vor der Verurteilung, und er kehrt reumütig zu seiner Frau, die mit der Suppe stumm in der Stube auf ihn wartet, zurück. In der ersten Rezension, übertitelt „Die Straße“, bezeichnet Kracauer den Film als „Meisterleistung“, der die Leere und Sinnlosigkeit der Straße und den dahinter lauernden Tod überzeugend dargestellt habe. Der Film stelle das ,,[...] Zusammentreffen von schmachtender Seele und existenzlosem Geschiebe [sic!] lautlos und schauerlich dar." (2004c [1924], S. 54) Die Menschen bewegten sich allesamt durch eine schemenhafte Welt. In der zweiten Rezension, übertitelt „Ein Film“, weist Kracauer auf das gezeigte, lediglich „,scheinhafte Leben“ hin, das sich in ,äußerlichen Begebenheiten“ erschöpfe und nur noch ,punktuelle Ereignisse" kenne (ebd., S. 56). Die zuvor als schematisch bezeichnete Welt wird nun zur ,zerfetzten Welt“. Er schreibt: „Keine Begegnung der Seelen findet statt, keine sinnvolle, dauernde Verknüpfung umklammert und bindet, nichts Tragisches zwischen ihnen geschieht, das ja zu seiner Voraussetzung eine konkrete Beziehung und in ihr gegründete wirkliche Entscheidungen hätte - nur Figuren stoßen zusammen, Ereignisse tragen sich zu und Situation reiht sich blind an Situation; das alles ohne Kontinuität und Folge, ein gespenstisches, unwirkliches Beisammen unwirklicher Menschen, das die leer fließende Zeit nicht zu erfüllen vermag. Der in die zerstückelte Welt versetzte Einzelne, der etwa ein Bewußtsein von sich selber hat, ist in ihr einsam schlechthin.“ (2004d, S. 57) Letztendlich drücke sich darin - so Kracauer in „Filmbild und Prophetenrede“ aus dem Jahr 1925 - die fehlende Glaubensgemeinschaft aus. Dort heißt es: „Das vor einiger Zeit in manchen deutschen 
Städten vorgeführte Filmwerk DIE STRASSE zeigt eine Welt, in der die Verknüpfung der Menschen mit dem Göttlichen ungetan bleibt. Diese Menschen der Großstadt haben keinen Bezug auf das Obere, sie sind nur noch ein solches Außen, wie die Straße selber es ist, auf der sich vieles begibt, ohne daß etwas geschieht. Das Getriebe der Figuren gleicht dem Wirbel der Atome, sie begegnen sich nicht, sondern prallen zusammen, sie treiben auseinander und trennen sich nicht.“ (2004e, S. 138).

Auffällig daran ist, dass Kracauer keine Religion - sei sie von jüdischer oder christlicher Herkunft - herausnimmt, sondern diese vielmehr übergreifend als eine Institution auffasst, und auch die Religionsausübung nicht als zeitgemäße Lebenspraxis anerkennt. Vielmehr macht er das Göttliche und die Glaubensgemeinschaft zu kritikalen Ordnungsbegriffen, weil sich die ziellos dahintreibenden Menschen davon entfernt zu haben scheinen.

Sosehr Kracauer dem Film - bei aller Kritik - auch positive Potenziale attestiert, so wenig sieht er sie in der Fotografie: Für ihn stellt sie die „kalte“ mediale Entsprechung einer sinnentleerten modernen Welt dar, auf deren säkularer Oberfläche nur noch die äußeren Hüllen der Dinge seelen- und leblos übrigblieben. Sie sei durch kühle Exaktheit bei der Aufzeichnung der vor ihrem Objektiv befindlichen, zeiträumlich situierten und punktuell zur Erscheinung gebrachten Objekte gekennzeichnet. Der wissenschaftlichen Exaktheit der Kamera stehe die entseelte Hülle der Dinge und Menschen gegenüber.

In seinem Essay aus dem Jahr 1927 stellt Kracauer die Fotografie beispielsweise als Resultat eines registrierenden, intentionslosen Apparates dem sinnhaft erinnernden, jedoch zeiträumlich wenig exakten lebendigen Gedächtnis gegenüber. Während die Fotografie sich um die übriggebliebenen, materialen äußeren Reste der Welt bemühe, sei das Gedächtnis sinnhaft strukturiert und an der Lebendigkeit der erinnerten Geschichte ausgerichtet. Während Erinnerung und Gedächtnis dabei Züge des gemeinschaftlichen Gedenkens trügen, sei die Fotografie lediglich technischer Ausdruck der auf der Ratio basierenden modernen Gesellschaft. Mit zunehmenden zeitlichen Abstand zur Aufnahme entweiche aus der Fotografie jedoch auch der letzte Rest ihrer Verlebendigung durch das sinnzuschreibende Gedächtnis, bis sie selbst als materiales Artefakt nur noch bloße Hülle, vergilbtes Papier, einen Naturrest darstelle: „Das photographische Archiv versammelt im Abbild die letzten Elemente der dem Gemeinten entfremdeten Natur.“ (1977e [1927], S. 38)4.

Jenseits dieser werkgeschichtlichen Weiterführung wird deutlich, wenn auch nicht von Kracauer expliziert, dass Medien, Technik und kollektive Gemeinschaft in einem Spannungsverhältnis zueinanderstehen, das sich nicht einfach auflösen lässt: Denn die reproduktive Technik ,entseelt“ die abgebildeten Dinge und Personen und überlässt sie ihrem Absterben, ihrem Tod, bis hin zur Verwesung durch materiales Verblassen ihrer Inhalte.

In seiner ideologiekritischen Arbeit zum Weimarer Film „Von Caligari zu Hitler“ (1984 [1947]) sind es dann vor allem die Dämonen, denen Kracauer filmhistorisch

\footnotetext{
4 Und, so könnte mit Blick auf seine Kritik an den modernen Wissenschaften ergänzt werden, es ist diese entleerte, abstrakte und übriggebliebene Natur, um die sich die Naturwissenschaft als Gegenstand bemüht, ohne darin den Menschen in seiner ganzen sinnhaften Lebendigkeit und dem Wunsch nach Transzendenz zu erkennen bzw. wiederzuerkennen.
} 
nachspürt. Er folgt dabei einem sozialpsychologischen Ansatz, da er in den Filmen tiefenpsychologische Dispositionen angelegt sieht, die den Aufstieg Adolf Hitlers vorwegnehmen: „Mittels Aufnahme der sichtbaren Welt - ob nun die gängige Realität oder ein imaginäres Universum - liefern Filme daher Schlüssel zu verborgenen geistigen Prozessen.“ (ebd., S. 13) In den von Kracauer besprochenen Filmen geistern diabolische Spukgestalten und besessene Figuren umher, ,[...] der deutsche Schauspieler [erwies sich] als zwielichtiger Zauberer, der die dämonischen Kräfte der menschlichen Natur beschwor." (ebd., S. 34) Der Homunculus in DER Golem, WIE ER IN DIE WELT KAM von Paul Wegener und Carl Boese (D 1920) ist Kracauer zufolge ein Film, der den Vorgriff auf Hitler und das Rachemotiv als Reaktion auf den Versailler Vertrag filmisch umsetzt (ebd., S. $38 \mathrm{f}$.).

In seinem zweiten großen Werk ,Theory of Film“, an dem er in den USA arbeitet und das 1960 auf Englisch erscheint, ${ }^{5}$ schreibt er im Vorwort, dass es sich beim Film um etwas grundlegend Menschliches, ,[...] eine Verhaltensweise zur Welt, eine Form menschlicher Existenz“ (1985 [1960], S. 14) handele. Derart macht er den Film zu einem exponierten Medium der Gesellschaft, indem er dessen produktive Potenziale auslotet. Menschliche Existenz finde ihren Ausdruck in einer ,,materialen Ästhetik“ des Films, die in der Lage sei, deren Vergänglichkeit aufzuheben: „Und da jedes Medium den Dingen besonders zugetan ist, die es allein darstellen kann, scheint das Kino vom Wunsch beseelt, vorübergleitendes materielles Leben festzuhalten, Leben in seiner vergänglichsten Form.“ (ebd., S. 11).

Kracauer ist sich bewusst, wie schon der Fotografie-Aufsatz zeigt, dass sowohl Fotografie als auch Film in einem ambivalenten Verhältnis zu den Entfremdungserfahrungen moderner Menschen stehen. Denn aufgrund ihrer jeweils spezifischen Zeitlichkeit sind beide Medien auf unterschiedliche Weise mit dem Tod und der Endlichkeit des Menschen verbunden (Mülder-Bach 2005, S. 854). ${ }^{6}$ Ja, beide führen ihm dies buchstäblich vor Augen. Dennoch sieht Kracauer paradoxerweise im Film ein Medium, dass diesen Zerfall (scheinbar) aufzufangen in der Lage ist.

Die individuelle Flucht ins Kino und die rezeptive Verschmelzung mit der Bilderwelt der filmischen Dinge sei das lustversprechende Surrogat für eine als verloren empfundene Sinnhaftigkeit und für den Verlust gemeinschaftlicher Ganzheit. Die individualisierten Zuschauer*innen seien ,Wartende“ in einer Zwischenwelt, die zwischen dem schönen Schein und der alles organisierenden, zweckentfremdeten Ratio der sie umgebenden Gesellschaft changiere. Die Zuschauer*innen machten die konkrete Erfahrung der ,last things before the last“ (so der englische Untertitel des letzten Geschichtswerks Kracauers) aufgrund der transzendierenden Wirkung des Filmischen, drängten so in den Vorraum der Geschichte ein, ohne zu den letzten Dingen hinter der Bilderwelt vorstoßen zu können.

\footnotetext{
5 Auch das Caligari-Buch erscheint 1947 in den USA auf Englisch. Die erste deutsche Übersetzung übernimmt 1958 der Rowohlt-Verlag. Allerdings wird die Studie um fast die Hälfte gekürzt, und die politischen Andeutungen werden herausgestrichen. Damit verlor diese erste Übersetzung die brisanten Stellen, die eine Anklage gegen die deutsche Gesellschaft darstellten (Brodersen 2001, S. 126ff.).

6 In den Nachbemerkungen zur ,Theorie des Films“ werden die Entwicklungen und Wendungen der Entstehung dieses Werks von Inka Mülder-Bach nachgezeichnet, vor allem das Verhältnis der „Marseiller Hefte“ als Skizzen zur „Theorie des Films“.
} 
Wie hat man sich dieses Surrogat vorzustellen? In der „Theorie des Films“ (1985 [1960]) entfällt die bis dahin ideologiekritisch-sozialpsychologische Perspektive aus verschiedenen Gründen. Dieses Werk, 1960 nach jahrzehntelanger Arbeit veröffentlicht, verfolgt eine „materiale Ästhetik“, wie Kracauer in der Einleitung zu diesem offenbar für ihn schwierigsten Werk schreibt. Der Film sei in der Lage, die uns umgebende Welt wiederzugeben und zu enthüllen. Er teile mit der Fotografie die Affinität zur äußeren Wirklichkeit. Der Film komme zu sich selber, sofern er der realistischen Tendenz folge und sich auf Inhalte fokussiere.

Demgegenüber lehnt Kracauer Filme als unfilmisch ab, sofern diese dem Impuls der formalistischen Tendenz nachgeben würden, d.h. die Technik der filmästhetischen Gestaltung über die inhaltliche Darstellung erheben, wie er es beispielsweise im Experimentalfilm - und, erstaunlicherweise, auch im Dokumentarfilm (Heinze 2018) - zu erkennen meint.

Zwar ist sich Kracauer durchaus bewusst, dass Filme zwangsläufig eine formalästhetische Gestaltung aufweisen, dass sie keineswegs Realität lediglich einfangen und abbilden können. Dennoch sucht er in ihnen nach solchen Darstellungsformaten und -weisen, von denen er annimmt, dass sie die äußere Wirklichkeit adäquat wiedergeben. Hierzu setzt er bei der Geschichte der Fotografie und ihrem realistischen Ursprung an; er beschäftigt sich mit den allgemeinen Merkmalen des Films und seiner Nähe zur ,ungestellten“ Realität; er dekonstruiert sämtliche Elemente und Bestandteile des Films; er differenziert unterschiedliche Filmformen und versucht, deren Eigenheiten nachzuspüren.

Eines der wegweisendsten Kapitel dieser Untersuchung stellt allerdings der Epilog mit dem Titel „Film in unserer Zeit“ dar. In ihm resümiert Kracauer die Bedeutung des Films als ein Medium, das in der Lage ist, dem Menschen die Welt, in der er lebt, konkret und als sinnliche Erfahrung vor Augen zu führen. Vorangestellt wird die Frage: „[W]elchen Wert hat die Erfahrung, die der Film vermittelt?“ (Kracauer 1985 [1960], S. 321) Einige seiner von Anbeginn seines Schaffens gewählten Formulierungen tauchen hier wieder auf, so wie die Rede vom „,Verlust der Innerlichkeit in der Moderne“ und vom „Verfall gemeinsamer Glaubensinhalte“ sowie vom „stetig wachsenden Prestige der exakten Wissenschaften“. Dafür durchquert er noch einmal die, wie er es nennt, „Ruinen alter Glaubensinhalte“, um im Liberalismus zu enden, der die Religion zur Privatsache werden lässt. Lapidar heißt es erneut: „Der Mensch unserer Gesellschaft ist ideologisch obdachlos.“ (ebd., S. 375).

Und erneut kritisiert Kracauer die modernen Wissenschaften, die als Ersatz für den Verlust des Glaubens aufträten und keine anderen Visionen als den technischen Fortschritt kennen würden. Durch den Abstraktionsgrad ihres Wissens würden sie die Menschen vom konkreten Erfahrungsreichtum, den sie im Verhältnis zu ihrer äußeren und inneren Welt entwickelt haben, abtrennen. Dieses Urteil fällt Kracauer auch und vor allem über die quantitativ verfahrenden Sozialwissenschaften. Derart schlage der Glauben an die technische Vernunft paradoxerweise in Esoterik um.

Ebenso kritisiert er aber auch die zeitgenössische Religion(en), die keine Antworten auf die Erscheinungen und Herausforderungen der Moderne zu geben in der Lage seien. Es gelte deshalb an die untersten Schichten der Realität (im Profanen) heranzukommen, um den Menschen wieder mit sich selbst und seiner Lebenswelt vertraut zu machen. Er schreibt: „Wenn wir uns aber der herrschenden Abstraktheit 
entledigen wollen, müssen wir vor allem diese materielle Dimension ins Auge fassen, die von der Wissenschaft erfolgreich vom Rest der Welt abgelöst worden ist. Denn wissenschaftliche und technologische Abstraktionen bedingen nachhaltig unser Denken; und sie alle verweisen uns auf physische Phänomene, während sie uns gleichzeitig von deren Qualitäten weglocken. Daher die Dringlichkeit, genau diese gegebenen und doch ungegebenen Phänomene in ihrer Konkretheit zu begreifen. Das wesentliche Material ,ästhetischer Wahrnehmung ' ist die physische Welt mit all dem was sie uns zu verstehen geben mag. Wir können nur dann darauf hoffen, der Realität nahezukommen, wenn wir ihre untersten Schichten durchdringen." (ebd., S. 387).

Film „durchforsche“ die physische Realität, er lege diese material „frei“ in einer niemals zuvor gesehenen Form, er mache uns wieder mit den vor unseren Augen liegenden Dingen vertraut, von denen wir uns entfernt haben (Kracauer 1985 [1960], S. $388 \mathrm{f}$.). Und dass dies nur dem Menschen gelingen kann, der zuvor entfremdet lebte, drückt sich in folgender beiläufigen Bemerkung aus: ,,[...] Und wir sind imstande, sie zu erfahren [die durch die Kamera aufgezeichnete Welt], weil wir fragmentarisch sind.“ (ebd. S. 389).

Hierzu merkt Kracauer an, dass wir jenseits der dargebotenen ,story“ eines Films in der Lage seien, einzelne Elemente durch unsere Wahrnehmung herauszulösen, etwa ein Gesicht, das Furcht oder Glück ausdrückt, eine Straße, die als Hintergrund der vordergründigen Szene wirkt usw. Unser eingeübtes Vermögen des fragmentarischen Blicks, mit dem wir Filme sehen, bringe uns in der Totalen oder im close-up die zutiefst menschlichen Züge unserer Lebenswelt nahe. Ein kurzer Augenblick, eine gekonnte Einstellung, eine beiläufige Zufälligkeit enthalte filmisch das Ganze des menschlichen Lebens: Das sei die große humane Leistung des Films.

Filme führten uns in die untersten materialen Schichten, in die Gegenständlichkeit des profanen Lebens - und hier kommt das eschatologische Moment, die Apotheose des Films zum Tragen -, sie bewegen sich, so Kracauer, ,von unten nach oben“ (ebd., S. 399); Filme steigen hinab zur materiellen Physis der Welt, um anschließend zu irgendeinem Problem oder zum Glauben aufzusteigen, freilich ohne diesen letztgültig erreichen zu können.

In diesem Bewegungsprozess kann der Film uns aber auch in den Zeugenstand rufen und Dinge vor Augen führen, die wir in der Realität nicht zu ertragen in der Lage sind. Diese Art des Spiegelns beschreibt Kracauer im berühmten Mythos-Kapitel „Das Haupt der Medusa“, das er angesichts der Nazi-Gräuel und der filmischen Aufzeichnungen in den KZs verfasste. Darin geht er davon aus, dass uns der Film Ereignisse aus der Realität in Erinnerung rufen kann, bei deren Ansicht wir ansonsten versteinern würden. Der Film wird zum errettenden Surrogat für etwas, das wir anders nicht begreifen können, gleichzeitig hilft er uns, so Kracauers optimistische Hoffnung, uns von den Übeln dieser Welt zu befreien (ebd., S. 395). Die Bewusstmachung der Welt erscheint als Transzendenzerfahrung im Film - zumindest traut Kracauer dem Film das Potenzial dazu grundsätzlich zu.

Während der Titel der englischen Fassung von „redemption“ (Erlösung) spricht, stimmte Kracauer in der deutschen Übersetzung dem Vorschlag Adornos zu, statt von Erlösung von „Errettung“ zu sprechen. Auch wenn Kracauer nach eigenem Bekunden hiermit auf eine bewusst theologische Bedeutung (Mülder-Bach 2005, 
S. 864) anspielen wollte, wird diese im Werk nicht weiter ausgearbeitet, sondern im Epilog lediglich angedeutet. So lässt er letztlich im Dunkeln, wovon der Film den Menschen erretten oder gar erlösen kann. Kracauer war sich offenbar der weitreichenden Bedeutung der Begriffsveränderung in der Übersetzung von ,redemption“ zu „Errettung“ nicht vollständig im Klaren, ebenso wenig, wohin diese begriffliche Unterscheidung das Werk bei strenger Auslegung beider Begriffe treiben könnte. Die religionslastigen Begrifflichkeiten ruft er hier nur noch als „,leere Signifikanten“ auf, als assoziative Anstöße, in die Leser*innen ihre eigenen Deutungen einschreiben müssen, während sie für ihn in ihrer affirmativen religiösen Bedeutung, die derartige Begriffe noch in seinem Frühwerk eingenommen haben, nicht mehr existieren.

Ganz weltlich ist Film für Kracauer ein Spiegel geworden, in dem sich der Mensch in seiner dinglichen und sozialen Umwelt in ihrer Konkretheit wiedererkennen kann, ein Spiegel, der zeigt, „was wir unter der Oberfläche über uns selber denken“ (1974 [1948], S. 249). Deshalb ermuntert uns Kracauer, in diesen Spiegel zu schauen.

\section{Schluss}

Angesichts der massiven Krisen, denen unser Planet und wir Menschen gegenwärtig ausgesetzt sind, angesichts der Tatsache, dass immer mehr „Wartende“ sich angesichts zuspitzender Krisen dazu entschließen, entweder auf die Seite der „Skeptiker“ zu wechseln, die ihren Skeptizismus so weit treiben, dass selbst die Lüge zur Wahrheit wird (,alternative Wahrheit“), oder als „Kurzschlussmenschen“ quasi religiösen, nationalistischen und faschistischen politischen Bewegungen oder religiös-sektiererischen Erweckungsbewegungen nachlaufen, also retardieren, bekommt Kracauers Position nicht nur etwas Emanzipatorisches, sondern zugleich Tröstendes. Sosehr er deutlich machen kann, dass es in der Moderne letztendlich keine Erlösung aus dem Wartezustand geben kann, weil ihr Zeitpfeil nicht auf die Vergangenheit, sondern die Zukunft gerichtet ist, auf ein Spiel mit Kontingenz statt Transzendenz, so sehr verweist er auch darauf, dass uns moderne Medien zwar nicht immer und unisono, aber des Öfteren eine Gelegenheit bieten, uns unserer Existenz zu vergegenwärtigen.

Diese Möglichkeit zur Selbstvergewisserung, die das mediale Spiegeln auszulösen vermag, löst er in zwei Richtungen auf: So arbeitet er heraus, dass die Spiegelung sowohl in Richtung einer psychosozialen Reflexion auf unser Selbst als auch in Richtung einer Bewusstwerdung derjenigen kollektiven gesellschaftlichen Bezüge verlaufen kann, in die wir unwillentlich eingebettet sind und die sich mithilfe unmittelbarer Erfahrung nicht länger begreifen lassen. Im Hinblick auf Letzteres nimmt Kracauer den von Niklas Luhmann an den Beginn seiner Medientheorie gestellten, fast schon lapidar zu nennenden Satz „Alles was wir über unsere Gesellschaft, ja über die Welt, in der wir leben, wissen, wissen wir durch die Massenmedien“ (Luhmann 2017, S. 9) aufgrund vielfältiger Belege vorweg.

Auch wenn Kracauer das Potenzial der Fotografie im Vergleich zum Film eher negativ einschätzt, lässt sich derweil auch in zeitgenössischer Fotografie die von ihm ausgemachte Vergegenwärtigungs- und Bewusstwerdungsfunktion entdecken. So führt uns beispielsweise der brasilianische Fotograf Sebastião Salgado mit ästhe- 
tisch „schönen“ Bildern die Destruktivität menschlicher Arbeit in den Goldminen Afrikas und Lateinamerikas vor, eine Arbeit, die gemacht werden muss, um die kapitalistische Ökonomie am Laufen zu halten und von der auch wir konsumistisch profitieren (Salgado et al. 1993; Salgado 2013). Von ihrer Zerstörungswut gegenüber der Natur und den arbeitenden Menschen wollen wir am liebsten die Augen verschließen, aber Salgados Fotografien zwingen uns, einen Blick in den Abgrund zu werfen.

Auch dem Film, man denke an Naturfilme, kritische Dokumentar- und Spielfilme oder Serien, aber auch Aktivist*innen-Filme auf YouTube und anderen Online-Plattformen, kann heutzutage eine wichtige Aufgabe und Wiedervergegenwärtigungsfunktion zukommen, sofern diese in der von Kracauer nahegelegten Weise wahrgenommen werden.

Vielleicht aber werden in einer gar nicht mehr allzu fernen Zukunft lediglich die Reste der äußeren Natur als filmische Realitätssimulation und Hülle erhalten bleiben und an das erinnern, was wir unwiederbringlich verloren haben. Verloren haben wir dann aber nicht den Glauben an etwas - ein Fokus, mit dem Kracauer sich ein Leben lang beschäftigt hat -, sondern die elementaren materiellen Voraussetzungen unserer physischen und psychischen Existenz.

Danksagung Ich danke Ulla Ralfs aus dem Fachbereich Sozialökonomie der Universität Hamburg für die kritische Durchsicht und sprachliche Überarbeitung dieses Beitrags.

Funding Open Access funding enabled and organized by Projekt DEAL.

Open Access Dieser Artikel wird unter der Creative Commons Namensnennung 4.0 International Lizenz veröffentlicht, welche die Nutzung, Vervielfältigung, Bearbeitung, Verbreitung und Wiedergabe in jeglichem Medium und Format erlaubt, sofern Sie den/die ursprünglichen Autor(en) und die Quelle ordnungsgemäß nennen, einen Link zur Creative Commons Lizenz beifügen und angeben, ob Änderungen vorgenommen wurden.

Die in diesem Artikel enthaltenen Bilder und sonstiges Drittmaterial unterliegen ebenfalls der genannten Creative Commons Lizenz, sofern sich aus der Abbildungslegende nichts anderes ergibt. Sofern das betreffende Material nicht unter der genannten Creative Commons Lizenz steht und die betreffende Handlung nicht nach gesetzlichen Vorschriften erlaubt ist, ist für die oben aufgeführten Weiterverwendungen des Materials die Einwilligung des jeweiligen Rechteinhabers einzuholen.

Weitere Details zur Lizenz entnehmen Sie bitte der Lizenzinformation auf http://creativecommons.org/ licenses/by/4.0/deed.de.

\section{Literatur}

Brodersen, Momme. 2001. Siegfried Kracauer. Reinbek: Rowohlt.

Burawoy, Michael. 2015. Public Sociology: Öffentliche Soziologie gegen Marktfundamentalismus und globale Ungleichheit. Weinheim, Basel: Beltz Juventa.

Butzer, Günter. 2009. MedienRevolution. Zum utopischen Diskurs in den Medientheorien Kracauers und Benjamins. In Denken durch die Dinge. Kracauer im Kontext, Hrsg. Frank Grunert, Dorothee Kimmich, 153-168. München: Wilhelm Fink.

Frisby, David. 1989. Fragmente der Moderne: Georg Simmel - Siegfried Kracauer - Walter Benjamin. Rheda-Wiedenbrück: Daedalus.

Frisby, David. 1990. Zwischen den Sphären. Siegfried Kracauer und der Detektivroman. In Siegfried Kracauer - Neue Interpretationen, Hrsg. Michael Kessler, Thomas Y. Levin, 39-58. Tübingen: Stauffenberg. 
Geertz, Clifford. 2015. Dichte Beschreibung: Beiträge zum Verstehen kultureller Systeme, 13. Aufl., Frankfurt am Main: Suhrkamp.

Heinze, Carsten. 2018. Siegfried Kracauer und der dokumentarische Film. In Der dokumentarische Film und die Wissenschaften, Hrsg. Carsten Heinze, Arthur Schlegelmilch, 7-58. Wiesbaden: Springer VS.

Jansen, Peter-Erwin, Christian Schmidt, und Martin Jay (Hrsg.). 2003. Leo Löwenthal - Siegfried Kracauer In steter Freundschaft: Briefwechsel 1921-1966. Springe: zu Klampen.

Koch, Gertrud. 2012. Siegfried Kracauer zur Einführung. Hamburg: Junius.

Kracauer, Siegfried. 1971. Soziologie als Wissenschaft. Frankfurt a. M.: Suhrkamp.

Kracauer, Siegfried. 1973. Geschichte - Vor den letzten Dingen. Frankfurt a. M.: Suhrkamp.

Kracauer, Siegfried. 1974. Filme mit einer Botschaft. In Siegfried Kracauer: Kino, Hrsg. Karsten Witte, 249-263. Frankfurt a. M.: Suhrkamp.

Kracauer, Siegfried. 1977a. Katholizismus und Relativismus. In Das Ornament der Masse: Essays, 187-196. Frankfurt a. M.: Suhrkamp.

Kracauer, Siegfried. 1977b. Die Wartenden. In Das Ornament der Masse: Essays, 106-119. Frankfurt a. M.: Suhrkamp.

Kracauer, Siegfried. 1977d. Kult der Zerstreuung. In Das Ornament der Masse: Essays, 311-317. Frankfurt a. M.: Suhrkamp.

Kracauer, Siegfried. 1977e. Die Photographie. In Das Ornament der Masse: Essays, 21-39. Frankfurt a. M.: Suhrkamp.

Kracauer, Siegfried. 1977f. Das Ornament der Masse. In Das Ornament der Masse: Essays, 50-63. Frankfurt a. M.: Suhrkamp.

Kracauer, Siegfried. 1984. Von Caligari zu Hitler. Eine psychologische Geschichte des deutschen Films. Frankfurt a. M.: Suhrkamp.

Kracauer, Siegfried. 1985. Theorie des Films. Die Errettung der äußeren Wirklichkeit. Frankfurt a. M.: Suhrkamp.

Kracauer, Siegfried. 1990a. Martin Buber. In Schriften 5.1 (Aufsätze 1915-1925), Hrsg. Inka Mülder-Bach, 236-242. Frankfurt a. M.: Suhrkamp.

Kracauer, Siegfried. 1990b. Zur religiösen Lage in Deutschland. In Schriften 5.1 (Aufsätze 1915-1925), Hrsg. Inka Mülder-Bach, 273-277. Frankfurt a. M.: Suhrkamp.

Kracauer, Siegfried. 2004a. Von der Erkenntnismöglichkeit seelischen Lebens. Eine Abhandlung [1916]. In Frühe Schriften aus dem Nachlaß, Bd. 9.1, Hrsg. Ingrid Belke, 121-168. Frankfurt a. M.: Suhrkamp.

Kracauer, Siegfried. 2004b. Die heilige Geschichte im Film [1916]. In Kleine Schriften zum Film, 1921-1927, Hrsg. Inka Mülder-Bach, 12-13. Frankfurt a. M.: Suhrkamp.

Kracauer, Siegfried. 2004c. Die Straße [1924]. In Kleine Schriften zum Film, 1921-1927, Hrsg. Inka Mülder-Bach, 54-56. Frankfurt a. M.: Suhrkamp.

Kracauer, Siegfried. 2004d. Ein Film [1924]. In Kleine Schriften zum Film, 1921-1927, Hrsg. Inka MülderBach, 56-58. Frankfurt a. M.: Suhrkamp.

Kracauer, Siegfried. 2004e. Filmbild und Prophetenrede [1925]. In Kleine Schriften zum Film, 1921-1927, Hrsg. Inka Mülder-Bach, 138-140. Frankfurt a. M.: Suhrkamp.

Kracauer, Siegfried. 2006. Der Detektiv - Roman. Werke, Bd. 1, 103-209. Frankfurt a. M.: Suhrkamp. Hrsg. Inka Mülder-Bach.

Kracauer, Siegfried. 2011. Autorität und Individualismus. In Essays, Feuilletons, Rezensionen, Bd. 5.1, Hrsg. Inka Mülder-Bach, 167-173. Frankfurt a. M.: Suhrkamp. 1906-1923.

Kracauer, Siegfried. 2017. Die Angestellten: Aus dem neuesten Deutschland, 15. Aufl., Frankfurt am Main: Suhrkamp.

Löwenthal, Leo. 2003. Wenn ich an Friedel denke: Rede anlässlich eines Kracauer - Symposions an der Columbia Universität in New York. In Leo Löwenthal - Siegfried Kracauer In steter Freundschaft: Briefwechsel 1921-1966, Hrsg. Peter-Erwin Jansen, Christian Schmidt, und Martin Jay, 268-282. Springe: zu Klampen.

Luhmann, Niklas. 2017. Die Realität der Massenmedien. Wiesbaden: Springer.

Mannheim, Karl. 1985. Ideologie und Utopie, 7. Aufl., Frankfurt am Main: Klostermann.

Matala de Mazza, Ethel. 2018. Der populäre Pakt: Verhandlungen der Moderne zwischen Operette und Feuilleton. Frankfurt am Main: S. Fischer.

Mitchell, William John Thomas. 2007. Picture theory: Essays on verbal and visual representation. Chicago: University of Chicago Press.

Mülder-Bach, Inka. 2005. Nachbemerkung und editorische Notiz. In Siegfried Kracauer: Theorie des Films, Hrsg. Inka Mülder-Bach, 847-874. Frankfurt a. M.: Suhrkamp.

Neun, Oliver. 2018. Öffentliche Soziologie. Baden-Baden: Nomos. 
Nowell-Smith, Geoffrey. 2006. Einleitung. In Geschichte des internationalen Films, Hrsg. Geoffrey Nowell-Smith, 3-5. Stuttgart, Weimar: J. B. Metzler.

Pollack, Detlef. 2018. Probleme der Definition von Religion. In Handbuch Religionssoziologie, Hrsg. Detlef Pollack, Volkhard Krech, Olaf Müller, und Markus Hero, 17-50. Wiesbaden: Springer VS.

Salgado, Sebastião. 2013. Genesis. Köln: Taschen.

Salgado, Sebastião, Eric Nepomuceno, und Lélia Wanick Salgado. 1993. Workers: an archaeology of the industrial age. New York: Aperture.

Später, Jörg. 2016. Siegfried Kracauer: Eine Biographie. Berlin: Suhrkamp.

Weber, Max. 1996. Wissenschaft als Beruf. Berlin: Duncker und Humblot.

Wendt, Victoria. 2010. Siegfried Kracauer - Einfluss und Wirken eines vermeintlichen Außenseiters der Weimarer Zeit. In Soziologie in Frankfurt: Eine Zwischenbilanz, Hrsg. Felicia Herrschaft, Klaus Lichtblau, 85-104. Wiesbaden: VS. 